\title{
CONF-850507--54
}

UCRL--91742

DE86 000020

\section{DIFFICULTIES WITH INFERRING NEUTRON CROSS SECTIONS FROM CHARGED-PARTICLE REACTIONS}

D. G. Gardner
M. A. Gardner

This paper was prepared for submittal to the International Conference on Nuclea. Data

for Basic and Applied Science

Santa Fe, NM, May 13-17, 1985

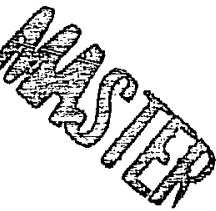

May 7,1985

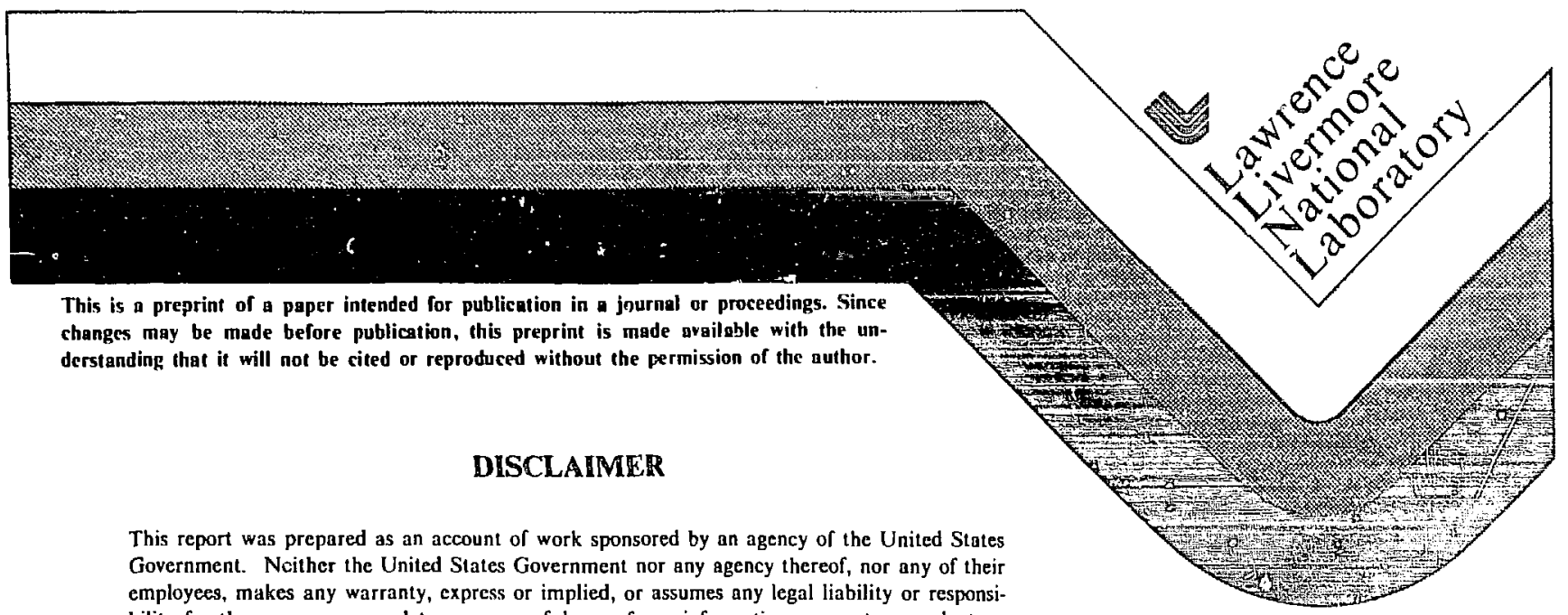
bility for the accuracy, completeness, or usefulness of any information, apparatus, product, or process disclosed, or represents that its use would not infringe privately owned rights. Reference herein to any specific commercial product, process, or service by trade name, trademark, manufacturer, or otherwise does not necessarily constitute or imply its endorsement, recommendation, or favoring by the United States Government or any agency thereof. The views and opinions of authors expressed herein do not neccssarily state or reflect those of the United States Government or any agency thereof. 


\title{
DIFFICULTIES WITH INFERRING NEUTRON CROSS SECTIONS FROM CHARGED-PARTICLE REACTION5 ${ }^{\circ}$
}

\author{
D. G. Gardner and M. A. Gardner \\ Lawrence Livermore National Laboratory, Livermore, CA 94550
}

\begin{abstract}
We have investigated problems associated with inferring cross sections for neutron reactions on unstable nuclei in the mass-90 region from charged-particle reactions on nearby stable targets. We cunclude that effects due to precompound evaporation, isospin, and multiple reaction paths severely limit the circumstances under which charged-particle studies may be directly and easily converted to neutron cross sections of useful accuracy.
\end{abstract}

\section{INTRODUCTION}

There are a number of areas of research in low-energy nuclear physics, such as reactor design and stellar nucleosynthesis, that require the knowledge of neutron reaction coss sections on unstable nuclei, which cannot be obtained through laboratory experiments. With modern computer codes, such cross sections may be calculated with coltidence if the nuclear information required as input to the calculation is well known. Charged-particle reactions on nearby stable targets may often yield some of this information: optical-model parameters to generate transmission coefficients, nuclear level densities, discrete nuclear level quantities, and gamma-ray strength functions, which may be directly related to the neutron reaction of interest.

Beyond supplying nuclear parameters, it would be highly desirable if the chargedparticle reaction information could itself be easily and directly converted into the desired neutron cross sections. For example, if we are interested in the reaction ${ }^{87} \mathrm{Y}(\mathrm{n}, \mathrm{p})^{87} \mathrm{Sr}$, we might attempt to derive the neutron cross sections from a study of the reaction ${ }^{87} \mathrm{Sr}\left(\mathrm{p}, \mathrm{p}^{\prime}\right)^{87} \mathrm{Sr}$ by using the relationship:

$$
\left.\sigma_{\mathrm{np}}\left({ }^{87} \mathrm{Y}\right)=\sigma_{\mathrm{pp}}{ }^{87} \mathrm{Sr}\right) \cdot \frac{\sigma_{\mathrm{n}}\left({ }^{88} \mathrm{Y}\right)^{*} \mathrm{CN}}{\sigma_{\mathrm{p}}\left({ }^{88} \mathrm{Y}\right)^{4} \mathrm{CN}} .
$$

Here $\sigma_{x}\left({ }^{88} Y{ }^{*} \mathrm{CN}\right.$ signifies the compound nucleus formation cross section of ${ }^{88} Y$ in its uistribution of excited states by the incident particle $x$ on the appropriate target nucleus, with due consideration of the effect of the separation energy of particle $x$ from ${ }^{88} Y$ on the choice of incident particle energy. The basis for this goes back to the work of S. N. Ghoshal, ${ }^{1}$ who showed that, under certain circumstances, the same excited compound nucleus formed by different reactions tended to decay by the formation of reaction products in ratios that were relatively independent of the initial formation reaction. To the extent that all of the reactions involved proceed statistically via compound nucleus reactions, and the initial compound nuclei all have the same distributions in energy, angular momentum, and parity, the basis of this approach is sound. In actual practice, the above constraints are never completely realized.

Let us assume that the incident neutron and proton energies are such that differences of distributions in energy and angular momentum may be ignored, that direct reactions do not contribute significantiy, and that multiple reaction paths leading to the same end product are not a probiem. Two remaining aspects must still be considered: differences in the precompound evaporation of protons and neutrons from the excited ${ }^{88} Y$ nucleus, and the

\footnotetext{
- Work performed under the auspices of the U.S. Department of Energy by the Lawrence Livermore National Laboratory under contract No. W-7405-ENG-48.
} 


\section{G. GARDNER AND M. A. GARDNER}

effect of isospin, which affects the proton-induced but not the neutron-induced reaction. Were it not for these last two "entrance channel" effects, Eq. (1) would provide an adequate approximation for incident particles in the 10-20 MeV energy region.

\section{PRECOMPOUND EVAPORATION}

A first requirement concerning reactions where precompound evaporation effects may be important is that the excited nucleus directly preceding the desired end product falls in tr"? same position in each reaction sequence. For example, the reaction sequence

${ }^{90} \mathrm{Zr}+\mathrm{t} \rightarrow\left[{ }^{93} \mathrm{Nb}\right] \rightarrow \alpha+\left[{ }^{89} \mathrm{Y}\right] \rightarrow \mathrm{p}+{ }^{88} \mathrm{Sr}$ is not an appropriate model for the reaction ${ }^{88} Y+n \rightarrow\left[{ }^{89} Y\right] \rightarrow P+{ }^{88}$ Sr because the first compound nucleus in each sequence is not the same. Proton evaporation from ${ }^{89} \mathrm{Y}$ in the triton reaction sequence is essentially without a precompound evaperation component, while that from ${ }^{89} \mathrm{Y}$ in the neutron reaction sequence may be predominantly precompound in nature for excitation energies in the $20-30 \mathrm{MeV}$ range. The energy spectrum for the evaporated protons differs greatly in shape and magnitude, and this can lead to large cross-section differences, even to differences in isomer populations should they be involved.

Where the incident particle is a nucleon, all else being equal, precompound evaporation of the incident type of nucleon is favored. In most cases, the incident nucleon scatters in the target nucleus to produce a 2-particle, 1-hole initial exciton state. Particle evaporation is favored over further scattering to higher-number particle-hole states when isospin is not a consideration. The probability that at least one of the two particles in the first exciton state is of the incident-particle type is large, and hence its re-evaporation is enhanced. This effect does not lend itself to a simple correction, because particle evaporation occurs from higher exciton states whose populations change with excitation energy. The effect of isospin is also to favor particle evaporation from certain types of higher exciton states, further contributing to a "softer" evaporated particle energy spectrum.

\section{ISOSPIN}

As an example, consider the reaction ${ }^{87} \mathrm{Sr}+\mathrm{p}$; our statistical model calculations proceed in the usual way, except for two aspects. First, each of the particle evaporation paths is weighted by the square of the appropriate isospin Clebsch-Gordan coefficient. ${ }^{2}$ The same factors are applied to both precompound and compound-nucleus evaporation processes. Then, we made two limiting cases of calculations: with isospin conserved $\left(\sigma^{>}, \mu=0\right)$ and with isospin completely damped $\left(\sigma^{<}, \mu=1\right)$. Finally, these two cases may be added together with the weighting factors

$$
\sigma=\frac{1-\mu}{2 T_{0}+1} \sigma^{>}+\frac{2 T_{0}+\mu}{2 T_{0}+1} \sigma^{<} .
$$

We include gamma-ray competition, but neglect isospin splitting of the giant dipole resonance. Although a damping fraction of $\mu=0.5$ is probably not a bad approximation for this mass region and for the energy renge of interest here, we will use $\mu=0$ and 1 in Eq. (2) to display the range of values sparined by the two limits.

\section{RESULTS}

Recently, the Los Alamos National Laboratory carried out experimental measurements of proton-induced reactions on isotopes of strontium; some of the preliminary results have been made available to us. ${ }^{3}$ In Fig. 1(a) and (b), we compare some of these data with calculations we have made using our 1979 nuclear parameter values ${ }^{4}$ for optical-model potentials, nuclear level densities, discreie nuclear levels and their gamma-ray branchings, ant the gamma-ray strength functions, without any additional adjustment. 

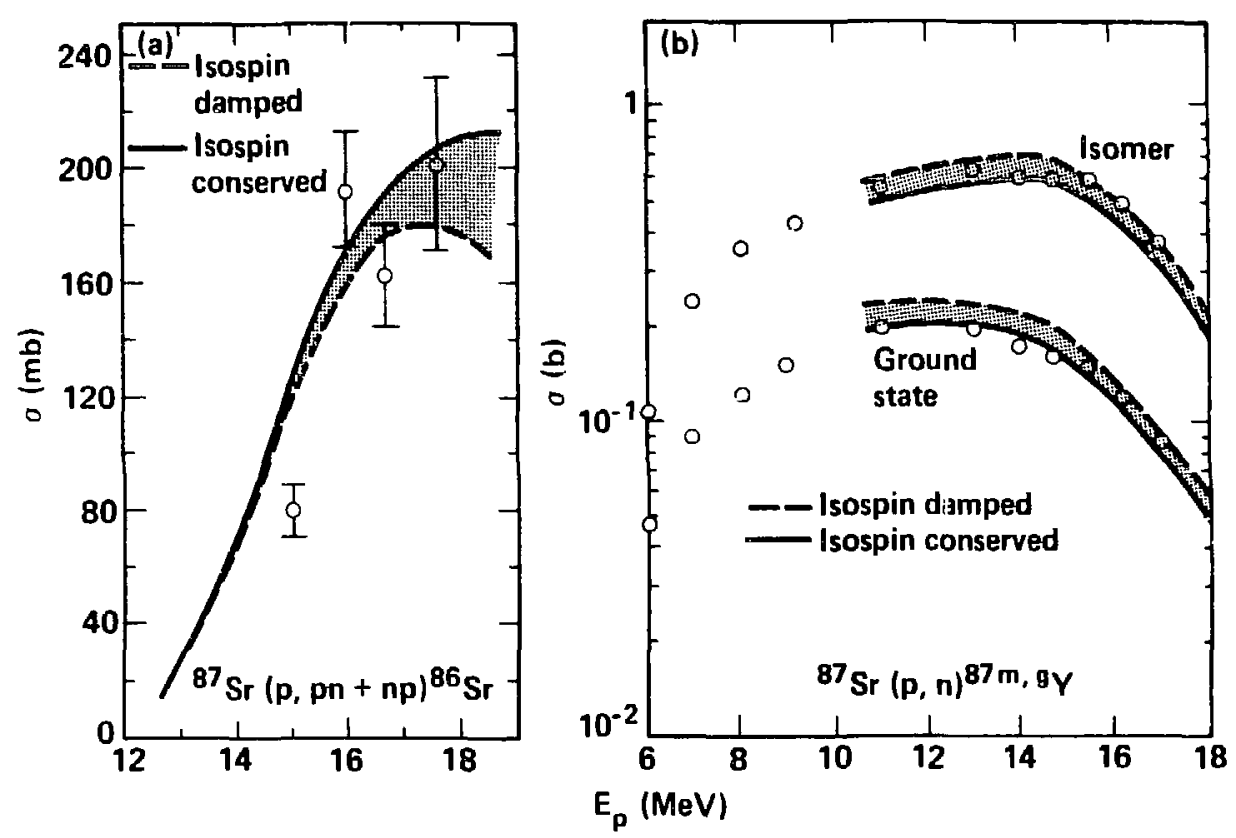

FIGURE 1. Comparison of experimental ${ }^{3}$ and calculated cross secticns for: (a) ${ }^{87} \mathrm{Sr}(\mathrm{p}, \mathrm{pn}+\mathrm{np})^{86} \mathrm{Sr}$, and (b) ${ }^{87} \mathrm{Sr}(\mathrm{p}, \mathrm{n})^{87 \mathrm{~m}, 8 \mathrm{Y}}$.

The usefulness of the Los Alamos proton reaction studies in validating our code calculations is quite apparent. However, we now demonstrate the problems involved in trying to convert them directly into reutron cross sections. A particularly poor situation is presented in Fig. 2(a). Here we show, as a function of incident particle energy, sets of calculated cross sections on ${ }^{87} \mathrm{Sr}$ for the reactions: $\left(p, p^{\prime}\right)$ vs $(n, p)$ and $(p, p n) v s(n, p n)$. Each cross section has been divided by the appropriate neutron or proton reaction cross section, yielding the ratio $\sigma_{\mathrm{x}} / \sigma_{\mathrm{xCN}}$ as described in Eq. (1). It is fair to use Eq. (1) because of our confidence in the neutron and proton optical models used to generate the respective reaction cross sections. We again show the two isospin-limiting cases for the proton-induced reactions; however, for a meaningful comparison with the neutron-induced results, one should visually average between the isospin-conserved and the isospin-damped extremes. As shown in Fig. 2(a), even without the uncertainty caused by the isospin damping factor, either neutron excitation function deduced from the proton calculations by multiplying by $\sigma_{\mathrm{nCN}}$ would be too high in general, and uncertain by as much as a factor of 2 or more. A somewhat improved situation is illustrated in Fig. 2(b), in which similar calculations for the ${ }^{87} \operatorname{Sr}(p, p n+n p)$ vs ${ }^{87} Y(n, p n+n p)$ cross sections are considered, with each cross section divided by the appropriate proton or neutron reaction cross section. Note: The energy scale for the proton reactions in Figs. 2(a) and (b) was shifted to account for the difference in neutron and proton separation energies from ${ }^{B 8} Y$. The isospin effect is reduced in the sum because it affects the $(p, p n)$ and $(p, n p)$ reactions in opposing directions. Nevertheless, the inferred neutron cross sections would still be in error by perhaps $50-100 \%$, and in the opposite direction from the situation depicted in Fig. 2(a). 


\section{G. GARDNER AND M. A. GARDNER}
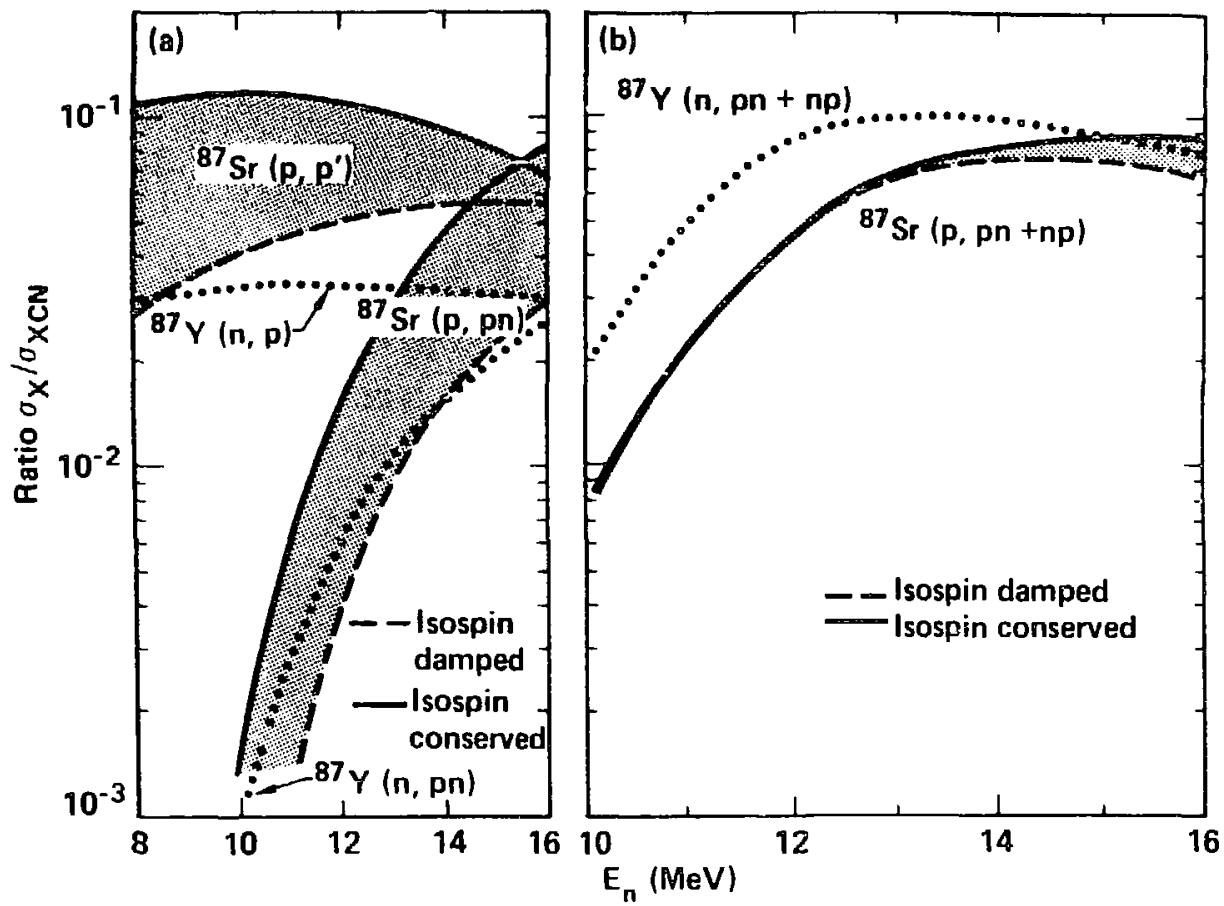

FIGURE 2. Calculated cross section ratios for selected ${ }^{87} \mathrm{~S} r+p$ and ${ }^{87} Y+n$ reactions.

Earlier we discussed the reaction ${ }^{90} \mathrm{Zr}(t, \alpha p)^{88} \mathrm{Sr}$, and pointed out the complications arising from prc ompound processes. We retum to this reaction now to illustrate how various reaction paihs ind reaction mechanisms also interfere with a direct conversion of one type of cross section into another type. If one assumes that the initial ${ }^{90} \mathrm{Zr}(t, \alpha){ }^{89} \mathrm{Y}$ reaction is predominantly a direct pick-up reaction, by measuring proton-alpha particles in coincidence, along with their respective energy spectra and angular distributions, one could infer both the excitation energy of the ${ }^{89} \mathrm{Y}$ nucleus that emitted protons and the ratio of the cross section for proton emission to that of forming the ${ }^{89} Y$ nucleus at some particular energy. However, it appears that the direct reaction path is small, compared with forming ${ }^{93} \mathrm{Nb}$ by triton capture and subsequent precompound and compound nucleus evaporation of protons and alpha particles. The protons are being emitted from two different nuclei, ${ }^{93} \mathrm{Nb}$ and ${ }^{89} \mathrm{Y}$, each having different energy and spin distributions, and when the energy of the system is sufficient that the product ${ }^{87} \mathrm{Sr}$ may be formed, a third source of protons appears to further complicate the situation.

\section{REFERENCES}

1. S. N. Ghoshal, Phys, Rev. 80, 939 (1950).

2. S. M. Grimes et al., Phys. Rev. C 5, 85 (1982).

3. D. W. Barr et al., "(p, $x n)$ Measurements on Strontium Isotopes," Los Alamos National Laboratory Nuclear Chemistry Division Annual Report FY82, LA-9797-PR (June 1983); J. C. Dousse et al., "Coincident Neutron-Proton Emission from Proton Bombardment of ${ }^{87} \mathrm{Sr}$ and ${ }^{91} \mathrm{Zr}$," to be published.

4. M. Gardner, "Statistical Model Calculations for the Zr Detector Cross Sections," Nuclear Chemistry Division Annual Report FY80, Lawrence Livermore National Laboratory, UCAR-10062-80/1 (1980), Pp. IV-A-16 to IV-A-19. 